\title{
DEPENDENCIA Y AUTONOMÍA DE LO SOCIAL EN LA TEORIAA SOCIAL: REPENSANDO LA CAUSALIDAD
}

\author{
DEPENDENCE AND AUTONOMY OF THE SOCIAL \\ IN SOCIAL THEORY: RETHINKING CAUSALITY
}

\section{ALVARO SÁEZ GARCÍA ${ }^{1}$}

\section{RESUMEN}

La independencia y la emergencia de la sociedad como una realidad autónoma suele ser un punto de partida común para integrarse a las filas de la ciencia social, en particular, de la sociología. En este artículo se sostiene que tal supuesto no es del todo correcto, por cuanto existen diversas formas y niveles a través de las cuales la sociedad manifiesta su dependencia. Se analiza en el presente artículo la dependencia particular que mantiene con el entorno material. Dicha dependencia es demostrada a través del análisis de las propias teorías presentes en las ciencias sociales, tomándose como muestra el trabajo teórico de tres de ellas: la sociología (Niklas Luhmann), la antropología (Marvin Harris) y la historia (Fernand Braudel $)^{2}$. La demostración se realiza a partir de la ampliación del concepto de causalidad y de su aplicación en las teorías mencionadas lo que permite concluir que la relación entre emergencia y causación es inversa. Ello permite observar con mayor claridad los límites a partir cuales la sociedad y otros sistemas pueden efectivamente autodefinirse como autónomos.

Palabras clave: Teoría social, causalidad, causación, emergencia, autonomía, entorno material.

${ }^{1}$ Licenciado en Sociología de la Universidad Alberto Hurtado. Profesor instructor del Departamento de Sociología de la Universidad Alberto Hurtado. Santiago, Chile. E-mail: asaez@ uahurtado.cl

${ }^{2}$ Esta breve selección de autores responde a los criterios de representatividad, interdisciplinariedad y a criterios prácticos de extensión. El de interdisciplinariedad exigía, a fin de poder referirse a "las ciencias sociales" con algún grado de verosimilitud, que las teorías pertenecieran a disciplinas diferentes. Como criterio de representatividad la selección fue hecha bajo el supuesto de que en el espectro de teorías que conforman las ciencias sociales ellas pueden dividirse entre aquellas que entregan mayor autonomía a lo social y aquellas que, por el contrario, enfatizan las 


\begin{abstract}
Independence and emergence of society as an autonomous reality tends to be a common starting point to integrate into social sciences, in particular, in sociology. This article argues that this assumption isn't entirely correct because there are different forms and levels in which society expresses its dependency. In this article we analyze the particular dependency that is maintained with the material environment. This dependency is shown through the analysis of some of social science's own theories, taking as examples the theoretical work of three disciplines: sociology (Niklas Luhmann), anthropology (Marvin Harris) and history (Fernand Braudel). The demonstration involves expanding the concept of causality and its application in the theories previously mentioned, thus allowing us to conclude that there is an inverse relationship between emergence and causation. This relationship permits a more clear observation of the boundaries from which society and other systems can effectively self-define themselves as autonomous.
\end{abstract}

Keywords: Social theory, causality, causation, autonomy, independence, material environment.

Recibido: 13.08.11. Aceptado: 30.03.12.

U NA DE LAS FORMAS de ejercer influencia es comunicar e investigar acerca de ella. En el presente artículo caracterizaremos las distintas formas en que la influencia, conceptualizada como causalidad se ejerce. Para ello nos detendremos en la relación particular de causalidades ejercidas entre dos niveles complejos: el entorno material y la sociedad. Observaremos los márgenes de los que depende y es posible la autonomía y la independencia de lo social. Recogiendo la división en niveles que componen el esquema AGIL en Parsons en el paradigma de la condición humana (Parsons, 1978) y la que realiza Emmeche en las teorías de la complejidad (Emmeche et al., 2000) diremos que es posible distinguir cuatro niveles generales de análisis: los niveles físico, biológico, psíquico y social. En función de tales niveles las ciencias orientan su investigación y definen sus diferentes objetos de análisis. Éstos se entrelazan ejerciéndose influencias recíprocas y posibilitándose mutuamente, siendo cada uno de ellos emergente con respecto al anterior. A estos cuatro niveles de complejidad, tácita o

condiciones materiales en las que ella se asienta. En relación a este último criterio, Luhmann fue seleccionado como un representante del primer grupo, Harris como representante del segundo y Braudel como un punto medio entre los dos polos. Considerando estos tres criterios de selección de la muestra se decidió excluir del análisis las teorías sociales de K. Marx, L. White, P. Bourdieu, T. Parsons, E. Durkheim, B. Moore y C. Geertz. 
implícitamente, se ha referido extensamente la literatura científica. Dentro del ámbito de las ciencias sociales se ha analizado con particular énfasis la vinculación entre los niveles físico-biológico (cerebro) y psíquico (mente) (Crane, 2001; Davidson, 1970; Edelman, 1987; Fodor, 1987; Stichs, 1983; Kim, 1993). La presente investigación se ocupa de la vinculación interniveles más distante y la menos explorada. Se interesa por la relación entre los niveles físico-biológico y el social. A fin de facilitar nuestra argumentación denominaremos en adelante a los dos primeros niveles como entorno material (ver Gráfico 1).

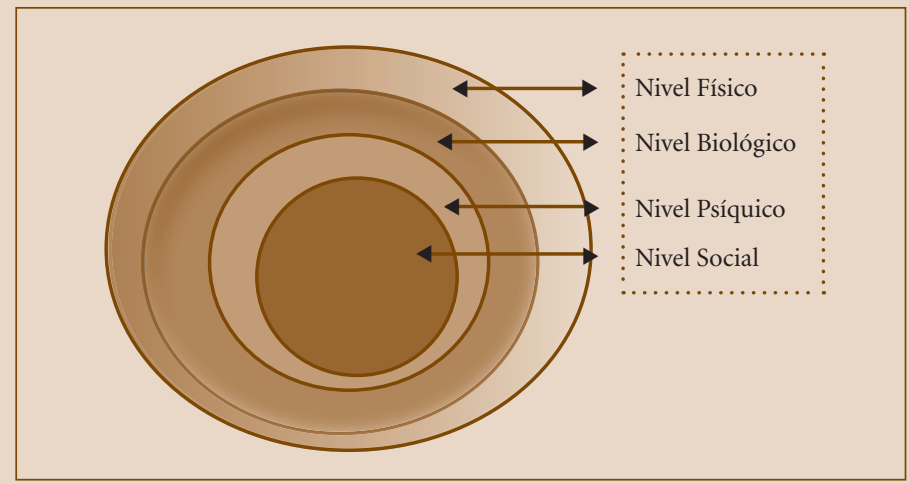

Gráfico 1.

Se ubica en los cimientos de la ciencia la premisa de que en el mundo real (social y físico) no reina el azar, pero que, sin embargo, tampoco se trata de una rígida y monótona estructura invariante. La ciencia en este escenario tiene una particular relación con el orden: lo observa, lo describe y lo crea. Una de las respuestas más antiguas a la pregunta por el orden se encuentra en la teoría de las causas de Aristóteles, quien indica cuatro causas que, interrelacionadas unas con otras, permitirían la concreción de todo efecto real. Dos causas del ser: la formal $y$ la material. Y dos causas del devenir: la eficiente y la final (Aristóteles, 2000). El principal inconveniente con esta conceptualización es que fue forjada teniendo como trasfondo objetos físicos y el resto de la tipología se construye desde ahí.

Otra respuesta más actual ofrece Bunge, quien explora las distintas formas en que este orden se configura en la ciencia, llamándolas: las categorías de determinación. Bunge identifica ocho categorías de determinación, cada 
una de las cuales da cuenta de diferentes formas de observar y describir el orden del mundo: las determinaciones causal, mecánica, estadística, estructural, teleológica, la interdependencia y las autodeterminaciones cuantitativa y cualitativa (Bunge, 1961: 30). Recogemos de Bunge la afirmación de que la determinación causal, la que usualmente es identificada simplemente como "causalidad", es sólo una entre otras formas de producción de orden y que ella no constituye el tipo de causalidad científica, pues ni siquiera parece ser la más frecuente ${ }^{3}$. Pero sus categorías son excesivas, además de no ser exhaustivas, pues los criterios de selección de las categorías varían de una a otra ${ }^{4}$. Proponemos seguir un camino diferente orientando los tipos de causación a través de un criterio único que englobe tales conceptualizaciones: el criterio de abstracción.

Al observar las causalidades tanto en Bunge como en Aristóteles, podemos distinguir entre formas más concretas de causalidad y otras más abstractas. Dado que la causalidad en general es una forma de producción de orden originada tanto desde el interior como desde el exterior de los fenómenos o sistemas, nuestra conceptualización para la causalidad se asienta en la distinción concreto/abstracto que da forma a la tipología, mientras que la distinción interno/externo se remite al contenido (ver Gráfico 2).

\begin{tabular}{|l|l|}
\hline $\begin{array}{l}\text { Ejemplo de otras formas de causación } \\
\text { subsumidas (Bunge/Aristóteles) }\end{array}$ & Tipos de causación \\
\hline & Absoluta \\
\hline Causal/ eficiente & Fuerte (en la actualidad) \\
\hline Teleológica/ formal & Media (en la posibilidad) \\
\hline Material/ cualitativa & Débil (en la condición de posibilidad) \\
\hline & Nula \\
\hline
\end{tabular}

Gráfico 2.

${ }^{3}$ Por ello, el trabajo de Emilie Durkheim para permitir que las regularidades sociales sean visibles e investigables empíricamente por una ciencia especializada como la sociología tropieza al dar el primer paso en la medida en que su hecho social está fuertemente asociado a esta forma de causalidad.

${ }^{4}$ Antecedente-consecuente para el caso de la determinación causal, medio-fines para la determinación teleológica, etc. El propio Bunge, por lo demás, está de acuerdo con nuestra afirmación al señalar que "si profundizáramos en nuestro análisis, seguramente hallaríamos otros tipos de determinación” (Bunge, 1961:31). 
En los extremos se ubican la causación absoluta y nula, que si bien no se encuentran en la realidad demarcan sus límites, permitiendo caracterizar debidamente el espectro de causación efectivo. Evitamos así caer en la descripción falaz, en la descripción de lo no-posible, al mismo tiempo nos permite contrarrestar el recurso retórico-bélico común de "estirar" los conceptos hasta el absurdo donde ninguna utilidad prestan a la argumentación (Schopenhauer, 1997: 41). En el horizonte de la causación efectiva la graduación de la distinción concreto/abstracto se caracteriza del siguiente modo.

La causación fuerte (ver Gráfico 3) se realiza en el marco de la actualidad y en ella el orden temporal de los sucesos o acontecimientos es determinante. Imaginemos un soldado alemán durante la Segunda Guerra Mundial que tras inmensas presiones y sufrimientos decide terminar con su vida suicidándose con un revólver en el campo de batalla. Decimos que estaba vivo "hasta" que lo alcanzó una bala, "luego" murió. En consecuencia, la bala externa y determinante fue la causa eficiente de su muerte. Este es el tipo de causación que usualmente se identifica como "la causa" científica, por ser el más directamente observable, controlable y medible (Hume, 1996), y desde luego es también la causa médica de su muerte. Esta forma de causación se remite al momento exacto y al acto de la selección de la posibilidad que puede realizarse al interior de un mismo sistema o, también, desde un sistema hacia (contra) otro sistema.

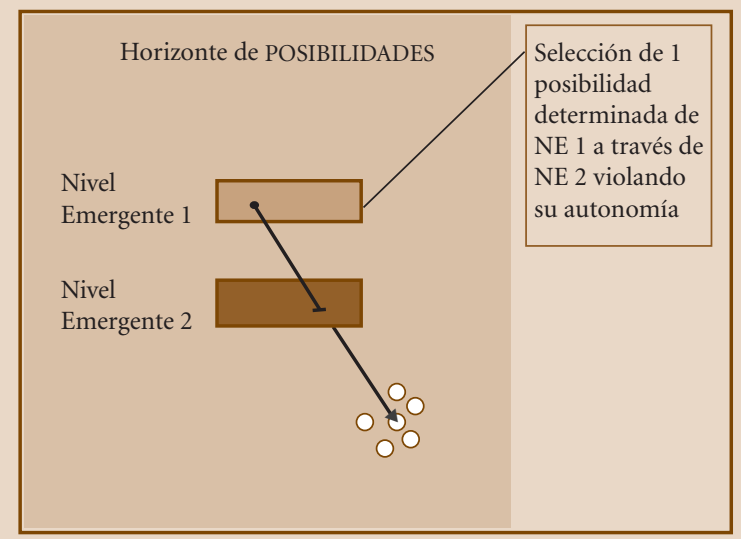

Gráfico 3. 
Ahora bien, ¿¿realmente la bala causó la muerte? Sí y no. La muerte en tanto acontecimiento pudo haber sido desencadenada por otros medios más o menos dolorosos, tanto por él mismo soldado, por sus cercanos y por sus enemigos en el campo de batalla desde luego. Es decir, había un horizonte de posibilidades que incluía el acontecimiento de una bala en la sien, plausibilizándolo, haciéndola viable. Por ello decimos que hay además una causación media (ver Gráfico 4). En primer lugar, resulta a todas luces improbable imaginar el desenlace fatal del soldado si éste, en vez de estar en un campo de batalla, hubiese estado en el campo cultivando hortalizas o leyendo un libro. En segundo lugar, en el campo de batalla pudo seleccionarse otras alternativas igualmente fatales dentro de un abanico de posibilidades prácticas, por ejemplo un suicidio altruista en el frente de batalla, un bombardeo aéreo, fuego cruzado, entre otras. Desde este punto de vista la causa efectiva es anecdótica, pues su muerte en realidad parece mayormente determinada por su contexto. De esta manera cobra sentido la idea de un espectro de posibilidades prácticas objetivas que restringe la selección de acontecimientos, pero que posibilita el acontecimiento (Emmeche et al., 2000: 9). Es evidente que en el campo de batalla no podía ocurrir "cualquier cosa", existía un marco determinado y estructurado que limitaba y hacía posible un número finito de desenlaces empíricos, tanto en lo que respecta a la vida de los soldados como en otros asuntos ${ }^{5}$.

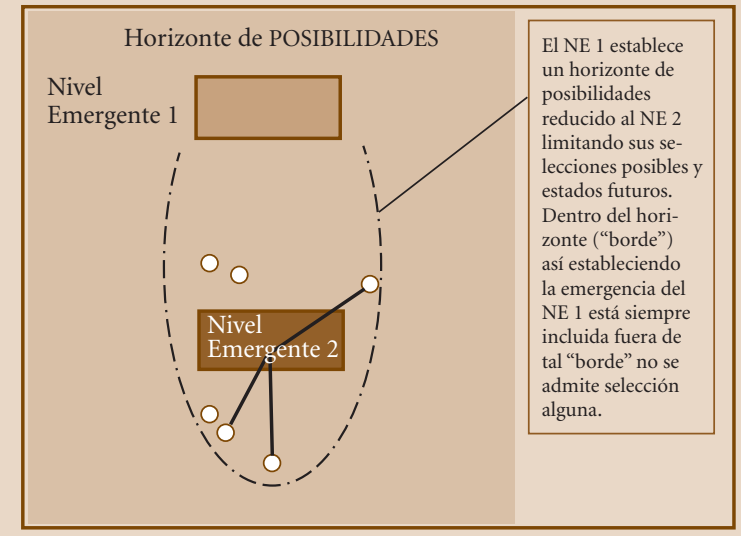

Gráfico 4.

\footnotetext{
${ }^{5} \mathrm{Si}$ ocurriera lo contrario, tanto el cine de ciencia-ficción hollywoodense como el realismo mágico en literatura, habrían sido rotundos fracasos sin ninguna novedad que ofrecer a la audiencia. Ahí sucede como real lo no-posible.
} 
Esta forma de causación podríamos definirla según su intensidad como una limitación estructural de lo posible (Mascareño, 2007, 2008), como una seducción de un sistema a otro o como una invitación a la autorregulación. Sin embargo, más importante que la definición específica que se privilegie es comprender que estas formas de causación media no obligan y, pese a ello (!), ejercen sin embargo una forma de influencia causal. Una que admite un mayor grado de autonomía del sistema que recibe la causación, desde luego, y en ello radican tanto su grandeza como su miseria, pues actúan soterradamente a espaldas del observador.

Si damos otro ligero paso a través de la abstracción, podemos observar que tal marco de posibilidades requiere de condiciones externas a sí mismo. A ello apunta el concepto de condición de posibilidad y la causalidad débil (ver Gráfico 5). Como sus términos lo indican, se sitúa más allá de la mera posibilidad y su disponibilidad en el tiempo para centrarse en sus condiciones. Fue Kant quien acuñó el término en su labor por demostrar que ciertas condiciones subjetivas del entendimiento, las categorías puras, podían tener validez objetiva pese a no tener contenido empírico, en la medida en que debían ser presupuestas para todo acto de conocer y que sin ellas, por tanto, ningún conocimiento era posible. Estableció así con claridad una diferencia entre el orden temporal de los fenómenos (causación fuerte) y el orden de la validez que recogemos en el siguiente extracto: "ningún conocimiento precede a la experiencia y todo conocimiento comienza con ella. (...) Pero, aunque todo nuestro conocimiento empieza con la experiencia, no por eso

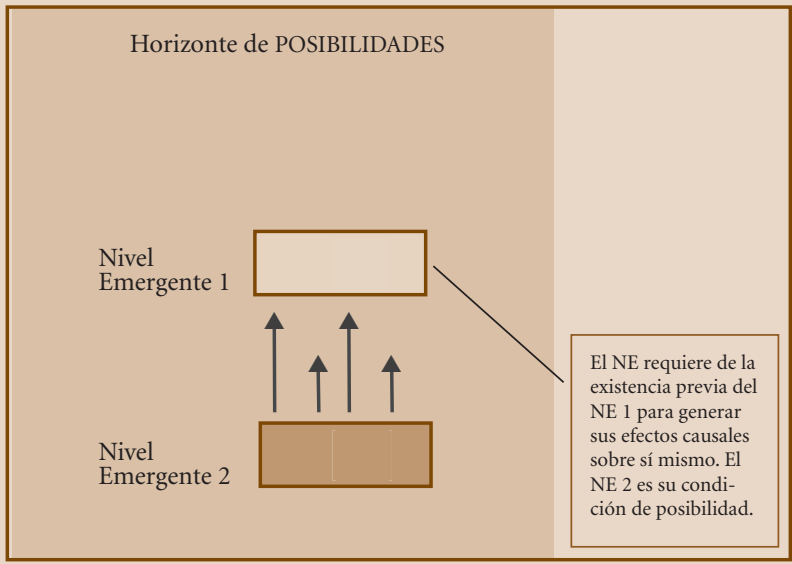

Gráfico 5. 
procede todo él de la experiencia" (Kant, 1998: 42). El entendimiento forja así sus distinciones y categorías con una libertad enorme, requiere únicamente de la experiencia el suministro de materia prima. La causación débil supone así los mayores grados de autonomía para los fenómenos. Ciertos fenómenos deben ser presupuestos como condición de posibilidad de otros, sin ellos su autonomía se viene al suelo.

¿Las balas causan la muerte? Nuevamente, sí y no. Era condición de posibilidad del suicidio del soldado la posición desfavorable y sin retorno en que se encontraba Alemania luego de años de guerra, económicamente extenuada y frente a una inminente derrota militar e ideológica. Condición de posibilidad de la emergencia de la sociedad es la existencia de seres humanos. Cuando los fenómenos son emergentes la causación interna desborda la causación externa, permitiendo sólo mínimos puntos de contacto e influencia (¡nunca menos!), pero donde la existencia previa de ese otro influyente debe quedar siempre presupuesta a fin de que el fenómeno autocausado pueda, de hecho, generar sus efectos causales sobre sí mismo. Se caracteriza por la contingencia en la medida en que el fenómeno causado dispone de una enorme libertad para determinar sus estados, sus procesos y sus efectos causales sobre sí mismo.

A partir de lo expuesto la relación entre causación y emergencia se transparenta. A medida que aumenta la causación externa, es decir, de un fenómeno o sistema sobre otro, disminuye la autonomía del que la recibe desfavoreciendo su emergencia. Por el contrario, cualquiera de las tres formas que adopte la causación interna o autocausación ella favorece la emergencia del fenómeno. Es en la relación de ambas fuerzas que se dirime el grado de emergencia en un momento dado. Así, la causación externa, que es de la que nos ocupamos principalmente en el presente artículo, favorece o lleva adosada determinadas formas de emergencia de manera inversamente proporcional (ver Gráfico 6).

\begin{tabular}{|c|l|}
\hline Causación externa & Emergencia \\
\hline Absoluta & No emerge \\
\hline Fuerte & Débil \\
\hline Media & Media \\
\hline Débil & Fuerte \\
\hline Nula & No emerge \\
\hline
\end{tabular}

Gráfico 6. 
La relación cerebro/mente ha sido profusamente analizada en los últimos años en relación al problema de la causación y la emergencia (Crane, 2001; Davidson, 1970; Edelman, 1987; Fodor, 1987; Stichs, 1983; Kim 1993) y su complejidad escapa a cualquier síntesis en estas páginas, pero de manera muy simplificada nos sirve como ilustración. Si decimos que todos los procesos mentales son seleccionados y coordinados por el cerebro, la mente no puede considerarse algo distinto de aquél y, en consecuencia, no podríamos hablar de otro orden, un orden emergente. Dado que la mente y sus características se reducirían a las del cerebro, la causación del cerebro hacia la mente sería absoluta. En breve: no hay mente autónoma. Ahora bien, si para argumentar la existencia de la mente como un fenómeno nuevo e irreductible (Stephan, 1999), es decir, emergente, nos apoyamos en el otro extremo estaremos defendiendo la causación nula y negaremos ahora la existencia del cerebro, lo que sería sin duda bastante menos afortunado. La respuesta debe, por tanto, situarse entre ambos extremos (Varela, 1983; 1988). El mismo razonamiento podemos utilizarlo por analogía para analizar la relación entre entorno material y sociedad que abordaremos en las teorías sociales a continuación.

\section{LA OSCILACIÓN DÉBIL-MEDIA DE LA CAUSACIÓN EN LA TEORÍA DE SISTEMAS}

Los sistemas emergen a partir de la diferencia entre sistema y entorno, afirma Niklas Luhmann. Es el sistema el que crea sus propios límites, sus propias estructuras y sus propias operaciones encontrándose, a la vez, clausurado y abierto (Luhmann, 2007: 73). Clausurado operativamente y abierto cognitivamente. La ácida controversia en torno a si los sistemas han de ser abiertos o cerrados es resuelta apelando a un retorsio argumenti (Schopenhauer, 1997), en la forma: precisamente y gracias a que el sistema está cerrado puede, entonces, y sólo entonces, estar abierto. No puede haber, por tanto, ninguna transferencia de información entre sistema y entorno (Luhmann, 1989: 18). Ningún input, ningún output: "No existe ninguna irritación que provenga del entorno del sistema, ni ninguna transferencia de irritación del entorno al sistema. Siempre se trata de una construcción propia del sistema, de una autoirritación -naturalmente que posterior a influjos provenientes del entorno" (Luhmann, 2007: 87).

Como lo que nos interesa demostrar aquí es la existencia de un tipo de relación de causalidad del entorno material hacia la sociedad, ¿cómo he- 
mos de comprender el entorno material en el esquema sistémico?, ¿cómo inciden, si es que lo hacen, esos "influjos" provenientes del entorno?

Luhmann aclara, a fin de evitar interpretaciones equívocas, que para comprender adecuadamente sus planteamientos de vocación paradigmática y universalista debemos considerar sus planteamientos como el de la diferencia entre sistema/entorno, que no es por tanto una teoría del sistema ${ }^{6}$. Señala que con la elección del término autopoiesis intenta tomar distancia del solipsismo constructivista y de la autohipóstasis (Luhmann, 1996: 27). La relación entre sistema y entorno sería así una relación constitutiva. El entorno no sería una categoría marginal al sistema, es decir, el sistema no sería ontológica, ni analíticamente más importante que el entorno (Luhmann, 1998: 172-173), ya que "el entorno siempre coactúa y (...) sin él nada -absolutamente nada- sucede" (Luhmann, 2007: 69).

Anunciado lo anterior no obstante Luhmann co-argumenta con las siguientes descripciones: "El entorno es un estado de cosas relativo al sistema. Cada sistema se delimita a sí mismo frente a su entorno (...) Por consiguiente, también la unidad del entorno está constituida por el sistema. $<$ El entorno $>$ es sólo un correlato negativo al sistema (...) El entorno es, simplemente, <todo lo demás>" (Luhmann, 1998: 176). O dicho quizás con mayor claridad aún: "La sociedad es un sistema determinado total y exclusivamente por sí mismo" (Luhmann, 2007: 70).

¿A qué se debe esta doble afirmación?, ¿se trata de una fatal contradicción o de una ingeniosa paradoja? Una respuesta factible es no considerarla como contradicción y abordarla en cambio como una paradoja para ir en busca de alguna distinción que permita "desplegar la paradoja de un modo productivo", haciéndola invisible y operativa a la vez. Esta es efectivamente una decisión teórica válida, pero no encontramos en Luhmann tal distinción articuladora. Todavía podría ser formulado como un retorsio argumenti como precisamente y gracias a que el sistema depende del entorno puede, entonces, $y$ sólo entonces, ser independiente de él, pero la radicalidad de la (o)posición anteriormente expuesta nos distancia de asumir tales estrategias retóricas. Afirmamos, en cambio, que en ocasiones la realidad impone al observador límites a su creatividad y productividad por más bellos, hábiles o ingeniosos que sean los malabares de la conceptualización teórica. El observador, al fin y al cabo, no es un mago (¿o sí?).

${ }^{6}$ Una discusión sobre los alcances de la teoría de sistemas como paradigma científico en Sáez (2012). Una discusión no luhmanniana sobre el universalismo en Chernilo (2007). 
Satisfechas por el entorno material las condiciones mínimas que requiere la sociedad, ella dispone de una flexibilidad y capacidad de autodeterminación inmensa. Por ello la causación del entorno material sobre la sociedad en comparación con la que recibe la sociedad de sus propios efectos autoproducidos es débil, y por ello, también, es que el entorno no obliga, ni ejerce por tanto una causación fuerte. Tal vez en las sociedades arcaicas donde la influencia del entorno material es ciertamente mayor, pero en la sociedad moderna el entorno material más bien motiva, seduce, invita.

Sin tales condiciones mínimas el sistema sociedad simplemente se derrumba. Si tales condiciones son perturbadas o peligran, ella se tambalea visibilizándolas de inmediato. Esto se observa con claridad en los desastres ambientales o en los períodos de escasez alimentaria, donde la independencia de la sociedad con respecto a su entorno material es repensada y puesta en tela de juicio cada vez que el riesgo de catástrofes globales y locales hace sentir su peso (¡causal!) sobre el sistema. El sistema necesita del entorno para autorreproducirse en el tiempo, por una parte, y éste al mismo tiempo seduce al sistema limitando sus posibilidades futuras. En ello consiste la oscilación causal del entorno material hacia la sociedad.

Por otra parte, como Luhmann está de acuerdo en que "en primer lugar lo que se necesita es un concepto de sociedad si quiere esclarecerse qué es el entorno visto desde ahí" (Luhmann, 2007: 96), su indicación inicial apunta a la sociedad, mientras que el entorno en general queda definido como ese "todo lo demás". Queda así determinado por oposición, lo que es un problema si lo que queremos observar precisamente es ese entorno material y sus posibles influencias ${ }^{7}$. Pese a autoidentificarse como una teoría fundada en la diferencia, al conceptualizar el entorno material lo hace refiriéndose a un continuum de materialidad, es decir, una continuidad indiferenciada y por tanto no observable desde la teoría.

Si quisiéramos realizar la indicación inicial en el entorno material tematizando a la sociedad como entorno de ese entorno material, tendríamos entonces, para hablar desde la teoría de sistemas, que indicar como sistema al entorno material. Tendríamos que indicarlo, quizás, como ecosistema. No obstante, para Luhmann ello sería apropiado "only if external boundaries could be supplied. But this is not the case. It does not even help to

\footnotetext{
${ }^{7}$ Algo similar ocurre cuando se estudia la historia, por ejemplo, mediante la utilización de los que nos hemos tomado la libertad de denominar como "conceptos-sombras", tales como el de sociedad tradicional de Durkheim, construidos simplemente como la contracara en negativo de la sociedad moderna.
} 
define systems by means of self-regulation instead of by boundaries" (Luhmann, 1989: 81). En consecuencia, la distinción sistema/entorno colapsa al ser aplicada sobre el entorno material como indicación directriz. En resumen, "la teoría de los sistemas autorreferenciales [no] sobrepasa la teoría causal" (Luhmann, 1998: 34) se instala, tal vez incómoda, en un extremo.

\section{LA OSCILACIÓN MEDIA-FUERTE DE LA CAUSACIÓN EN LA TEORÍA DEL DETERMINISMO INFRAESTRUCTURAL}

Bajo el concepto de infraestructura, Marvin Harris incluye, tanto los medios o modos de producción como los de reproducción social. Los primeros abarcan a la tecnología y al entorno material o natural. Harris busca definir la cantidad de energía que dispone un determinado sistema sociocultural para, posteriormente, orientar la investigación hacia cómo se utiliza y consume tal energía. De esta forma, la cantidad y eficiencia energética en la producción y el consumo se transforman en aspectos centrales. El entorno material es en este esquema la fuente única de energía. La tecnología "solo" media en este traspaso, permitiendo disponer de aquélla en menor o mayor grado y, por esa razón, es que las sociedades industriales, aun las más desarrolladas técnicamente, no se libran de la influencia del ambiente (Harris, 1991: 270).

Por medios de reproducción social, en cambio, Harris considera la reproducción humana, esto es: la producción de niños. El límite a la cantidad de energía que se puede extraer de un determinado ecosistema fija el límite de humanos que pueden vivir en él. En este sentido, la presión demográfica es considerada fundamental en la argumentación antropológica de Harris. Hasta tal punto, que la considerara la causa principal de la expansión histórica de las fuerzas productivas (Harris, 1987: 82).

La infraestructura asimismo tiene una condición estratégica, "representa la principal zona interfacial entre naturaleza y cultura, la región fronteriza en la que se produce la interacción de las restricciones ecológicas, químicas y físicas a que está sujeta la acción humana con las principales prácticas socioculturales destinadas a intentar superar o modificar dichas restricciones" (Harris, 1987: 73). Como Harris encuentra en la naturaleza la principal fuente de regularidades, la elección de esta zona resulta estratégica para la búsqueda de causalidades en el mundo social. Ello se basa "en que los hombres no pueden cambiar estas leyes. Lo más que podemos hacer es buscar un equilibrio entre la reproducción y la producción y el consumo 
de energía" (Harris, 1987: 73). De este modo, la necesidad de alimentación o los impulsos sexuales son universales, pero sus consecuencias y vías de satisfacción varían considerablemente.

Sobre la infraestructura se asientan las que Marx denominó relaciones de producción. La estructura comprende aspectos como el intercambio, la vida doméstica, el control y división del trabajo, y la propiedad económicolegal sobre la tecnología y los recursos naturales (Harris, 1991:310). Aquí el interés es dirigido hacia aquellas actividades mediante las cuales la sociedad distribuye los bienes materiales y simbólicos, por lo que se concluye que es el lugar en que Harris sitúa a la economía y a la política. Finalmente, sobre esta estructura descansa una superestructura social que acoge al arte, la religión y la personalidad de los individuos, pero al ser consideradas secundarias en comparación a la importancia explicativa de la infraestructura no son descritas acuciosamente en su obra.

¿Pertenece el determinismo infraestructural a las ciencias biofísicas? El nombre de Teoría del Determinismo Infraestructural se justifica en la premisa de que el éxito relativo de las innovaciones causales producidas en los tres componentes de los sistemas socioculturales mencionados (infraestructura, estructura y superestructura) es desigual. Como "no hay aspecto de la producción más importante que (...) la producción de seres humanos" (Harris, 1987: 87), la probabilidad de que una modificación en la infraestructura provoque efectos causales amplificadores de la desviación en las relaciones de producción y en el nivel político e ideológico es más frecuente que a la inversa, pues poseen grados disímiles de efectividad funcional.

La causación de la infraestructura que, como dijimos, contiene al entorno material, se limita entonces a restringir la disponibilidad de posibilidades presentes en los dos niveles superiores. Por ello, que una modificación en la conciencia social o en un movimiento social provoque cambios duraderos en los modos de producción y reproducción hasta el punto de alcanzar la estasis social es mucho menos probable que un aumento demográfico genere presiones duraderas sobre la estructura organizacional de la economía. Esa razón probabilística le permite afirmar, por ejemplo, que "no puede decirse que la lucha político-ideológica de las mujeres fuera la causa de los grandes cambios en la tecnología, la producción, la demanda de mano de obra barata, el surgimiento de las ciudades, el incremento de los costos de la crianza de los hijos..." (Harris, 1987: 90).

Algunos fenómenos de masas como la moda hippies, hip hop o algunas de más reciente aparición como la moda pokemón en Chile pueden 
considerarse así como expresión de la normalización de las "desviaciones" provenientes de la superestructura, reduciendo sus posibles efectos causales distorsionadores sobre la infraestructura al ser compatibilizadas, invisibilizadas y transadas en el mercado como una entre otras mercancías ${ }^{8}$.

En conclusión, la teoría del determinismo infraestructural no afirma, por consiguiente, que la estructura y superestructura sean un mero reflejo de la infraestructura (Harris, 1987: 88), una respuesta automática y en ese sentido su denominación es desafortunada. Ambas poseen un cierto grado de autonomía, señala Harris, pero esa autonomía está supeditada al espectro de posibilidades que la infraestructura (im)pone a su disposición (Harris, 1987: 88). Esta forma de primacía funcional (Harris, 1981: 205) es el eje de su propuesta teórica y es la que nos lleva a caracterizar, en primera instancia, el tipo de causación como una causación media.

Sin embargo, al mismo tiempo Harris atribuye a ciertas constantes naturales un papel preponderante en la explicación y descripción de los fenómenos sociales que analiza. El énfasis que otorga a la transformación (o generación) y distribución de energía desde el entorno material a la sociedad balancea a Harris en una cuerda extremadamente floja. Cuando cae, lamentablemente no lo hace siempre para el mismo lado. El adjetivo "cultural" de su materialismo está ahí, recogiendo la herencia de L. White, para defender que la causalidad de los fenómenos socioculturales se debe buscar en los propios fenómenos socioculturales y no en los determinismos de índole orgánica o inorgánica (Harris, 1987: 14). Sin embargo, cuando describe empíricamente algunos de ellos tales como el amor a las vacas de los hindúes o la repulsión hacia los cerdos por parte de algunas religiones ancestrales, el argumento se centra a ratos justamente en constantes naturales (Harris, 1987: 26, 43, 45). El que llamaremos "argumento termodinámico", basado en las leyes del mismo nombre, señala que existe un cierto orden en la generación y distribución de energía y éste, al ser utilizado en algunos pasajes para explicar fenómenos socioculturales, socava fuertemente la autonomía de la argumentación antropológica, contradiciendo sorpresivamente, y esto es válido también para L. White, el supuesto epistemológico inicial de que lo social sólo ha de explicarse por lo social.

Los detractores del materialismo cultural encuentran así justificación de peso, ya que efectivamente en ocasiones Harris cede a los encantos de la

\footnotetext{
${ }^{8}$ En Chile, el éxito de esta forma de "subversión rentable" se puede encontrar, por ejemplo, en la programación de farándula o también en pasquines humorísticos como The Clinic, donde se observan ambos procesos.
} 
termodinámica y cae en un determinismo vacuo en el cual lo social pierde toda capacidad de autodeterminación y autorregulación. Estas dos lecturas sobre la causación desde la infraestructura hacia los niveles superiores nos permiten hablar de una oscilación media-fuerte en la teoría del determinismo infraestructural de Harris.

\section{LA LARGA DURACIÓN COMO CAUSACIÓN MEDIA}

Para Fernand Braudel, la Historia como disciplina ha estudiado fundamentalmente hitos políticos y militares y ha operado encantada por los "grandes hombres" y los "grandes acontecimientos" que, en una primera mirada, aparecen como las principales y únicas fuerzas motoras de la historia. Frente a esta forma de hacer historia, Braudel (pro)opone una forma de hacer historia, social y económica que bautiza como historia estructural. Ésta sería la encargada de describir el vasto universo de fenómenos de larga duración que escapan y rodean a las grandes guerras, a la voluntad de un rey o a los poderosos efectos iluminadores de algún líder espiritual.

En este esquema cobra relevancia el concepto de duración como medida de influencia para la Historia haciendo que la amplitud de perspectivas que tradicionalmente había caracterizado a la Historia se amplíe aun más. En palabras de Braudel: "La historia, si cabe decirlo así, es uno de los oficios menos estructurados de la ciencia social, y por lo tanto uno de los más flexibles, de los más abiertos" (Braudel, 1984: 117). Esto le permite traspasar las barreras disciplinarias como ninguna otra disciplina, pues "se encuentra en todos los lugares del banquete" (Braudel, 1984: 16) no hay ningún rincón del pantagruélico festín científico que le sea indiferente a sus febriles apetitos.

Como propuesta teórica, la dialéctica de la duración de Braudel consiste en la división del tiempo social en infinidad de tiempos que a modo de síntesis resume en tres: corta, media y larga duración. Tomando distancia con la llamada historia tradicional o de los acontecimientos, Braudel la caracteriza como excesivamente ocupada en lo anecdótico y lo efímero, excesivamente atenta al individuo. A este tiempo efervescente y dinámico se aboca el periodismo de crónica y la historia de corto alcance, aunque de fácil comprensión. La predilección por este tiempo social no se reduce exclusivamente a la historia, también los economistas, demógrafos, sociólogos, psicólogos e incluso geógrafos se encuentran inmersos en él: “Todas las ciencias sociales incurren en este error" (Braudel, 1984: 77). Una batalla, 
una opinión, un acto ceremonioso o una decisión política pueden ser algunos ejemplos de fenómenos de corta duración.

Sobre ellos se elevan los fenómenos de duración media, aquéllos donde la regularidad se hace visible y previsible. Es el tiempo de los ciclos y los semiciclos, un tiempo que permite explicar y comprender los fenómenos de menor duración. Inflaciones de ocho o nueve años, recesiones de cinco, hambrunas de medio siglo, migraciones, recurrentes fenómenos climáticos o creencias prolongadas son fenómenos que permanecen en el tiempo ejerciendo su influencia más allá del fugaz chispazo del acontecimiento. Son todos observables, no obstante, en el tiempo de los hombres, dentro del tiempo que dura la vida de un individuo.

Finalmente, los más importante para Braudel son los fenómenos de larga duración, un tiempo que silencioso atraviesa sociedades, culturas y civilizaciones sin ser percibido. Es un tiempo social evidente en la medida en que siempre está presente. Frente a nuestros ojos inyecta imperceptiblemente y en dosis ínfimas su influencia sedante en la conciencia cotidiana. Tan presente se encuentra que se hace invisible. Se funde y desaparece por su cotidianeidad. Su observación exige un gran esfuerzo de distanciamiento analítico, un extrañamiento.

La larga duración se conforma de regularidades extensas que tardan enormemente en desgastarse y nunca lo hacen abruptamente. La mejor descripción de este tiempo social es la que hace el propio historiador:

Ciertas estructuras están dotadas de tan larga vida que se convierten en elementos estables de una infinidad de relaciones: obstruyen la historia, la entorpecen y, por tanto, determinan su transcurrir. Otras, por el contrario, se desintegran más rápidamente. Pero todas ellas constituyen, al mismo tiempo, sostenes y obstáculos. En tanto que obstáculos, se presentan como límites (...) de los que el hombre y sus experiencias no pueden emanciparse. Piénsese en la dificultad de romper ciertos marcos geográficos, ciertas realidades biológicas, ciertos límites de la productividad, $\mathrm{y}$ hasta determinadas coacciones mentales representan prisiones de larga duración (...) El hombre es prisionero (...) de los climas, de las vegetaciones, de las poblaciones, de las culturas, de un equilibrio lentamente construido del que no puede apartarse sin correr el riesgo de volverlo a poner todo en tela de juicio (Braudel, 1984: 70).

A pesar de que pueden encontrarse fenómenos de larga duración en otros niveles, Braudel utiliza como su principal ejemplo la influencia que ejerce la geografía en las sociedades humanas. De hecho, tal como lo mencionábamos unos párrafos atrás, se ocupa del entorno material en toda la 
primera parte de El Mediterráneo..., situando allí los márgenes y características biofísicas en las cuales la vida de los hombres tiene lugar, aunque Braudel toma distancia del fatalismo geográfico de Ratzel, que por esos años gozaba de cierta reputación. Dice: "La geografía no explica toda la vida, ni toda la historia del hombre. Por muy importante que sea el escenario en que vive (el escenario queda y el hombre pasa), no lo gobierna todo. Fuera de sus dominios, no cesa de crecer el trabajo del hombre sobre las cosas y el del hombre sobre el hombre" (Braudel, 1953: 324), es decir, a nuestros fines, la causación fuerte queda de plano descartada. Braudel no admite esa intensidad de la constricción geográfica sobre la sociedad. Por otro lado, sin embargo, el hecho de situar al tiempo geográfico dentro de la duración que a juicio de Braudel es la principal (la larga), nos obliga a no descartar de plano la influencia de la geografía y a profundizar en sus alcances.

El ejemplo del efecto geopolítico y económico que ejerció la insularidad de Inglaterra nos es útil para resolver la pregunta por cuál es el tipo de causalidad que Braudel está dispuesto a otorgar a la geografía, en tanto como fenómeno de larga duración, sobre la sociedad. Veamos, Braudel sostiene que

la insularidad ayudó a Inglaterra a separarse de los demás países y a liberarse del capitalismo extranjero. Esto se consiguió fácilmente frente a Amberes (...) a los hanseáticos (...) a Ámsterdam (...) Por esa época [siglo XVIII] Ámsterdam domina lo esencial del comercio europeo. Pero Inglaterra contaba frente a ella con un medio de presión: los veleros holandeses, necesitaban hacer escala constantemente en puertos ingleses. Es, sin duda, esto lo que explica que Holanda haya aceptado de Inglaterra medidas proteccionistas que no aceptó de nadie más" (Braudel, 1985: 116).

Comprendamos debidamente los alcances y la (c)rudeza de la argumentación. Inglaterra disponía frente a Holanda de un poderoso medio de presión que le permitiría nada menos que resistir los embates del capitalismo holandés y que, posteriormente, le facilitaría a Londres el dominio incontestable de la economía-mundo europea a través de su Revolución Industrial ¿Cuál era esa poderosísima arma? ¡Su geografía!

La geografía en Braudel ejerce una fuerza constrictiva frente a la cual el hombre logra desembarazarse constantemente, pero dentro de un marco de posibilidades que el hombre no crea. De más estará decir que Ámsterdam hubiese deseado no perder su preponderancia económica en Europa en el siglo XVIII en favor de Londres, pero la selección de esa posibilidad 
escapaba a sus dominios, estaba mediada en mayor o menor grado por la geografía, ejerciendo una limitación estructural a las posibilidades de expansión de su capitalismo. Por esta razón, el entorno material identificado en la obra de Braudel con la geografía ejerce, en consecuencia, una causación media sobre la sociedad.

\section{REFERENCIAS}

Aristóteles (2000). Metafísica. Madrid: Gredos.

Braudel, F. (1953). El Mediterráneo y el mundo mediterráneo en la época de Felipe II. México: Fondo de Cultura Económica.

- (1984). Civilización material, economía y capitalismo, siglos XV-XVIII. Madrid: Alianza.

- (1985). La dinámica del capitalismo. Madrid: Alianza.

Bunge, M. (1961). Causalidad. El principio de causalidad en la ciencia moderna. Buenos Aires: Eudeba.

Chernilo, D. (2007). "A quest for universalism: Re-assessing the nature of classical social theory's cosmopolitanism", European Journal of Social Theory 10 (1): 17-35.

Crane, T. (2001). "The significance of emergence", en B. \&. Grant, Physicalism and its Discontents (pp. 207-224). Cambridge: Cambridge University Press.

Davidson, D. (1970). "Mental Events", in L. Foster and J. Swanson (eds.), Experience and Theory (pp. 79-101). London: Duckworth.

Edelman, G. (1987). The Remembered Present: a biological theory of consciousness. New York: Basic Books.

Emmeche, C. K.; Koppe, S. and Stjernfelt, F. (2000). "Levels, emergence and three versions of downward causation", en C. K. Emmeche, Downward Causation. Minds, Bodies and Matter (pp. 13-33). Aarhus: Aarhus University Press.

Fodor, J. (1987). Psychosemantics: the problem of meaning in the philosophy of mind. Massachusetts: Brad Books / MIT Press.

Harris, M. (1981). El desarrollo de la teoría antropológica. Madrid: Siglo XXI.

- (1987). El materialismo cultural. Madrid: Alianza.

(1991). Introducción a la antropología general. Madrid: Alianza.

Hume, D. (1996). Investigación sobre el conocimiento humano. Madrid: Alianza.

Kant, I. (1998). Crítica de la razón pura. Madrid: Alfaguara.

Kim, J. (1993). Supervenience and Mind. Cambridge: University Press.

Luhmann, N. (1989). Ecological Communication. Cambridge: University of Chicago.

(1996). La ciencia de la sociedad. México D.F.: Universidad Iberoamericana.

(1998). Sistemas sociales. España: Antrophos. (2007). Sociedad de la sociedad. México: Herder. 
Mascareño, A. (2007). "Emergencia y downward causation en la sociología sistémica”, en D. I. Cosmelli, Nuevos enfoques de la cognición: Redescubriendo la dinámica de la acción, la intención y la intersubjetividad. Santiago: Universidad Diego Portales.

(2008). "Comunication and Cognition: the Social Beyond Lenguage, Interaction and Culture". Integr Psych Behav 42, 200-207.

Parsons, T. (1978). Action Theory and the Human Condition. New York: The Free Press.

Sáez, A. (2012). “¿Sociología sistémica o paradigma sistémico?”, en H. Cadenas, A. Mascareño y A. Urquiza (eds.), Niklas Luhmann y el legado universalista de su teoría. Santiago: RIL Editores.

Schopenhauer, A. (1997). Dialéctica erística o el arte de tener la razón. Madrid: Trotta.

Stephan, A. (1999). "Varieties of Emergentism". Evolution and Cognition, 5.

Stichs, S. (1983). From Folk Psychology to Cognitive Science. Massachusetts: Bradford Books/MIT Press.

Varela, F. (1983). "Living Ways of Sense Making: A Middle Way Approach to Neuroscience", en P. Livingstone, Order and Disorder. Stanford: Anma Libris. (1988) [1984]. Conocer. Barcelona: Gedisa. 